Original Research Paper

\title{
An Efficient Course Resolution Multiple-Receiver Approach for Reception of Slow Fading Signals in Digital Wireless Communication Systems
}

\author{
Ashraf Mamdouh Aziz, Senior Member, IEEE, \\ Mohamed Aly Abdel-Rahman and Saeed Ahmed Al-Ghamdi \\ Department of Electrical Engineering, Al Baha University, AlBaha, Saudi Arabia
}

\author{
Article history \\ Received: $13-03-2015$ \\ Revised: 26-04-2015 \\ Accepted: 15-07-2015 \\ Corresponding Author: \\ Ashraf Mamdouh Aziz \\ Electrical Engineering \\ Department, AlBaha \\ University, AlBaha, Saudi \\ Arabia \\ Email: amaziz64@ieee.org
}

\begin{abstract}
Multiple-receiver digital wireless communication systems employ several receivers and a digital central receiver with data fusion to reduce the effect of multipath fading problems. The multiple receiver observations are processed at individual receivers and sent to a digital central receiver. The digital central receiver fuses all the individual receiver data to form global data on which symbol was transmitted. In this study, an efficient course resolution multiple-receiver approach for reception of slow fading signals in digital wireless communication systems is proposed. Each individual receiver observation is represented by course resolution data rather than high resolution data. The proposed approach is investigated for the case of no coherent frequency-shift keying in slow Rayleigh fading and additive Gaussian noise. The performance of the proposed approach is evaluated and compared to that of the optimum low resolution and high resolution approaches. Simulation results indicate that the proposed course resolution approach is efficient and cost effective. The results also indicate that the proposed approach can be used instead of high resolution approach without noticeable performance degradation which highly simplifies the construction and design of the digital wireless communication systems.
\end{abstract}

Keywords: Multiple Receivers Systems, Multipath Fading, Data Fusion, Course Resolution

\section{Introduction}

Multipath fading is caused whenever radio signal arrives at the receiver by more than one path. Characterization of communication channels that have randomly time-variant impulse responses serves as a model for fading digital communication signal transmission over many radio channels such as shortwave ionospheric radio communication (HF), tropospheric scatter (beyond-the horizon) radio communications (UHF and SHF) and ionospheric forward scatter (VHF). The time-variant impulse responses of these channels are due to the constantly changing physical characteristics of the media. Diversity techniques are used to provide replicas of the same data signal transmitted over independently fading channels (Aziz, 2011a; Chen and Tellambura, 2004; Simon and Alouini, 2005; Sendonaris et al., 2007). In this case, the probability that all the signal components will fade simultaneously is reduced. There are several ways to provide the communication system with $n$ independent fading replicas of the same databearing signal. One of the commonly used methods for achieving diversity techniques employs a single transmitting receiver and multiple receiving receivers. Such techniques are expected to increase the system performance compared to a single-receiver system. In the multiple-receiver diversity approach, the $j$ th receiver output, $j=1,2, \ldots . ., n$, is a multiple-bit information $u_{j}$ based only on the observations available at the corresponding receiver. The information from the $n$ communication receivers, $u_{1}, u_{2}, \ldots, u_{n}$, are then combined in a digital receiver using diversity combining to derive a global information. This is equivalent to a decision system with data fusion (Kot and Leung, 2003; Aziz, 2014a; 2014b).

There are three main methods for processing of multipath fading signals in multiple-receiver wireless 
communication systems (Viswanathan and Varshney, 1997; Mirjalily et al., 2003; Aziz, 2009a; 2014c). In the first method, the receivers produce samples with enough bits of resolution and the entire system will closely resemble the analog receiver diversity implementations. This method is called high resolution approach. Theoretically, the high resolution processing achieves the optimum performance at the expense of cost and complexity ( $\mathrm{Hu}$ and Blum, 2002; Blum, 1999; Willett et al., 2000; Aziz, 2013). This case includes the majority research in diversity techniques. However, using course resolution (only a few bits), instead of high resolution, could reduce cost and system complexity considerably. This is the principles of the second and the third methods of multipath fading signal processing. The second method is called course resolution approach where some preliminary processing of observations is carried out at each individual receiver and the results are then sent, using more than one-bit resolution, to the central receiver that combines the received data into final global data. This is not the case for the multiple-receiver diversity systems suggested by most of the published studies ( $\mathrm{Hu}$ and Blum, 2002; Blum, 1999). The third method is a special case of the second method where only one-bit resolution is used. The problem of one-bit resolution, which is also called low resolution data fusion, has been considered in many literatures (Viswanathan and Varshney, 1997; Mirjalily et al., 2003). The result is a loss of performance in low resolution (one-bit resolution) systems as compared to course resolution (few bits resolution) and high resolution (large number of bits resolution) approaches. Clearly, the advantages of the second and the third methods are reduced cost and complexity.

The optimum solution of multiple-receiver diversity systems in case of course resolution, even for the case of two bits per decision, is very complicated since it requires optimum quantization and the derivatives of the functional relationships between the error rates and the thresholds for all sensors (Kot and Leung, 2003; Viswanathan and Varshney, 1997). Thus the optimum analytical solution is not possible (Wen and Riteey, 1994; Tse et al., 2004; Aziz et al., 2011; 2011b). Some simplified structures based on one bit of quality information in addition to the receiver decisions are developed in the expense of a noticeable lower performance (Mirjalily et al., 2003) and (Wen and Riteey, 1994) for examples). Other simplified structures based on suboptimum objective functions are developed and can be used only in case of two bits per decision (Tse et al., 2004; Chau and Sun, 1996; Shin and Lee, 2008). The purpose of this paper is to introduce a simple, cost effective and efficient course resolution multiplereceiver approach. Unlike the significant contributions reported in the literatures, the proposed approach can easily be applied in case of any number of bits/decision and in case of non-identical sensors. It does not require analytic expressions for the derivatives of the functional relationships between the error rates and the thresholds for all sensors. The remainder of this paper is organized as follows. The optimum low resolution data fusion in wireless communication systems is briefly reviewed in section 2. Section 3 proposes a course resolution multiple-receiver approach. Section 4 compares the performance of the proposed approach and the low and the high resolution approaches in case of slow Rayleigh fading and additive Gaussian noise. The performance is determined as error rate performance for digital communication systems in fading and noise. Section 5 contains conclusion.

\section{Optimum Low Resolution Data Fusion in Wireless Communication Systems}

Since the proposed course resolution approach is based on binary wireless digital communication systems, a quick review of the optimal low resolution data fusion is presented in this section. In case of low resolution wireless communication systems, we are interested in discriminating between two message symbols 0 and 1 , encoded as two known waveforms $s_{0}(t)$ and $s_{1}(t)$. We suppose that we are to process a received signal $r(t)$ in additive noise $n(t)$. This is a binary hypothesis testing problem with two hypotheses; $H_{0}$ designating bit 0 and $H_{1}$ designating bit 1 , i.e.:

$H_{0}: r(t)=s_{0}(t)+n(t)$,
$H_{1}: r(t)=s_{1}(t)+n(t)$

We assume that there are $n$ local receivers with statistically independent observations $r_{1}, r_{2}, \ldots \ldots, r_{n}$ and have known probability distributions under both hypotheses $f_{R}\left(r_{i} \mid s_{0}\right)$ and $f_{R}\left(r_{i} \mid s_{1}\right), 1,2, \ldots, n$. It is also assumed that the observation at the $i^{\text {th }}$ receiver is a scalar $r_{i}$. The $i^{\text {th }}$ receiver output, $i=1,2, \ldots \ldots, n$, is a binary bit decision $u_{i}$ based only on the observations available at the corresponding receiver.

For each individual local receiver, the optimum structure should calculate the likelihood ratio and compare it to a likelihood threshold (Sendonaris et al., 2007; $\mathrm{Hu}$ and Blum, 2002). The optimal decision rule at each local receiver in case of low resolution can be described as:

$u_{i}=\left\{\begin{array}{l}1, \text { if } L R_{i}=\frac{f_{R}\left(r_{i} \mid s_{1}\right)}{f_{R}\left(r_{i} \mid s_{0}\right)} \geq t h_{i} \\ 0, \quad \text { otherwise, }\end{array}\right.$

where, $L R_{i}$ is the likelihood ratio at the $i^{\text {th }}$ receiver and the receiver's threshold, $t h_{i}$, is depending on the criterion of optimality. When the receiver Signal to 
Noise Ratio (SNR) estimates are available and the receiver SNR's change so slowly such that the SNR's estimates can be sent to the central receiver with very high precision, the conditional probability distributions in (2) can be replaced by $f_{R}\left(r_{i} \mid s_{0}, \gamma_{i}\right)$ and $f_{R}\left(r_{i} \mid s_{1}, \gamma_{i}\right), i=1,2, \ldots, n$, where $\gamma_{i}$ is the SNR estimate at receiver $i$ (Mirjalily et al., 2003).

The binary decisions from the $n$ communication receivers, $u_{l}, u_{2}, \ldots . ., u_{n}$, are then sent to a central receiver to derive a global decision $\hat{s}$ on which symbol was transmitted. According to the minimum probability of error rate criterion, the optimal decision combining rule for equally likely message bits (ones and zeros equally likely) is the Maximum Likelihood (ML) decision rule, namely $\hat{s}=1$ is chosen if (Sendonaris et al., 2007; Aziz, 2014b):

$$
\operatorname{Pr}\left(u_{1}, u_{2}, \ldots \ldots \ldots, u_{n} \mid s_{1}\right)>\operatorname{Pr}\left(u_{1}, u_{2}, \ldots \ldots \ldots, u_{n} \mid s_{0}\right)
$$

The ratio $\frac{\operatorname{Pr}\left(u_{1}, u_{2}, \ldots \ldots \ldots, u_{n} \mid s_{1}\right)}{\operatorname{Pr}\left(u_{1}, u_{2}, \ldots \ldots \ldots ., u_{n} \mid s_{0}\right)}$ is called the likelihood ratio of the set of the individual receiver decisions. By assuming the case of independent receiver observations, the optimal decision rule reduces to:

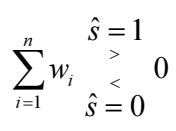

where, the coefficients $w_{i}, i=1,2, \ldots ., n$, are given in terms of the probabilities of correct decision $\left(P_{c i}\right)$ and the probabilities of bit error $\left(P_{e i}\right)$ as:

$$
w_{i}=\left\{\begin{array}{l}
\ln \left(\frac{P_{c i / 1}}{P_{e i / 0}}\right), \text { if } u_{i}=1 \\
\ln \left(\frac{P_{e i / 1}}{P_{c i / 0}}\right), \text { if } u_{i}=0, i=1,2, \ldots \ldots, n
\end{array}\right.
$$

$P_{c i / 1}=\operatorname{Pr}\left(u_{i}=k \mid s_{k}\right)$,

$P_{e i / 0}=\operatorname{Pr}\left(u_{i}=1-k \mid s_{k}\right), k=0,1$

The optimum fusion rule of the course resolution approach (4) is interpreted as the sum of the reliabilities of the receiver decisions. The final global decision of the digital central receiver is based on the sign of this sum.

\section{Proposed Course Resolution Multiple- Receiver Approach}

Consider a multipath fading environment where noncoherent binary Frequency-Shift Keying (FSK) is to be employed. In this case, $n$ individual receivers, each with its own receiver, are employed to achieve a diversity gain (Simon and Alouini, 2005; Hu and Blum, 2002; Willett et al., 2000). Each individual receiver employs the structure of the standard single-receiver noncoherent FSK receiver whose implementation is well known (Aziz, 2014c; Tse et al., 2004; Chau and Sun, 1996). Each of the $n$ individual receivers consists of two bandpass filters, each operated at a sinusoid with a different frequency $\left(\omega_{0}\right.$ or $\left.\omega_{1}\right)$. As shown in Fig. 1, the bandpass filters are operated at the sinusoid frequencies over the data period (bit interval). Frequency $\omega_{1}$ corresponds to a " 1 " being sent, while frequency $\omega_{0}$ corresponds to a " 0 " being sent. The outputs of the two bandpass filters are envelope detected and then sampled at the end of the bit period. The samples outputs from the two envelope detectors are the random variables $R_{0 j}$ and $R_{l j}$, which are the outputs of the $j$ th individual receiver. The data at each individual receiver is obtained by observing the value $R_{l j}-R_{0 j}$. The important value of the random variable $V_{j}=R_{l j}-R_{0 j}$ is denoted by $v_{j}$. The $j$ th sensor decision will be based on $v_{j}$ and on the signal-tonoise $(S N R)$ estimate (if it is available). Let $\Gamma_{j}$ be a random variable which denotes the $S N R$ estimate at sensor $j$ and let $\gamma_{j}$ denotes the samples of this random variable. The time-varying responses that occur and the characterization of the communication fading channels are treated in statistical terms (Shin and Lee, 2008). There are several probability distributions that can be considered in attempting to model the statistical characteristics of the fading channel. In case of large number of scatters in the channel, as the case in ionospheric or tropospheric signal propagation, central limit theorem leads to a Gaussian process model for the channel impulse response. If the process has zero mean, then the envelope of the channel response at any time instant has a Rayleigh probability distribution and the phase is uniformly distributed in the interval $\left(0, \pi_{2}\right)$ (Simon and Alouini, 2005; Kot and Leung, 2003).

In this study, slow Rayleigh fading is considered. The observations at each receiver are assumed to include additive zero-mean Gaussian noise. The fading and noise are assumed to be independent from receiver to receiver. In order to obtain signals that fade independently, certain separation is required between any two receivers (Simon and Alouini, 2005; Blum, 1999). Thus the multiple receivers must be spaced sufficiently far apart that the multipath components in the signal have significantly different propagation delays at the receivers. The two main steps for developing the proposed course resolution multiplereceiver approach are (1) obtaining the individual receiver likelihood ratios and (2) deriving the combining fusion rule of the digital central receiver. 


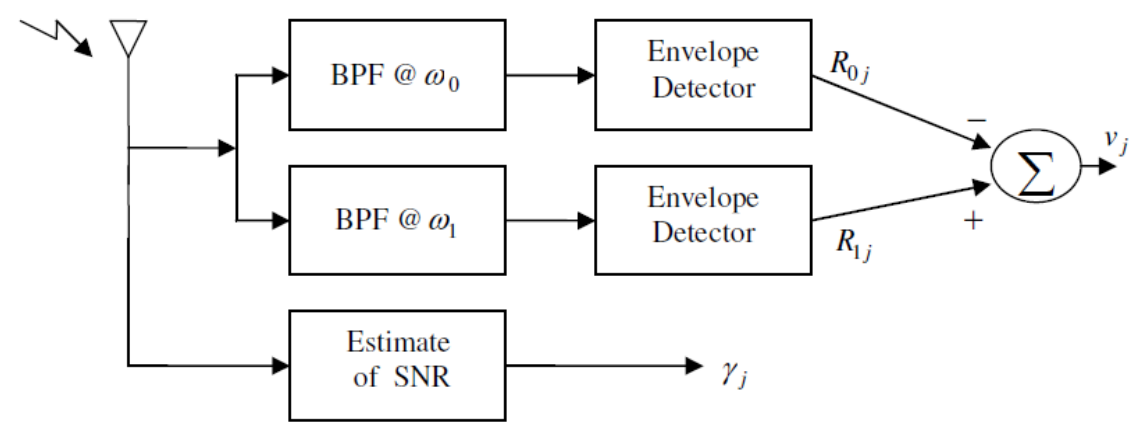

Fig. 1. Block diagram of each individual receiver in case of noncoherent FSK

\section{Obtaining the Individual Receiver Likelihood Ratios}

Since the optimum decision approach of the $j$ th individual receiver should perform a mapping of its likelihood ratio, which is a function of $V_{j}$ and $\Gamma_{j}$ then the value of $u_{j}$ will depend on the likelihood ratio. By assuming that the marginal Probability Density Function (PDF) of each receiver $S N R$ is the same under either hypothesis, we can write:

$\ln \frac{f v_{j}, \Gamma_{j}\left(v_{j}, \gamma_{j} \mid 1 \text { sent }\right)}{f v_{j}, \Gamma_{j}\left(v_{j}, \gamma_{j} \mid 0 \text { sent }\right)}=\ln \frac{f v_{j}\left(v_{j} \mid 1 \text { sent }, \gamma_{j}\right)}{f v_{j}\left(v_{j} \mid 0 \text { sent }, \gamma_{j}\right)}$

where, $f v_{j}, \Gamma_{j}\left(v_{j}, \gamma_{j} \mid s\right.$ sent $)$ is the joint $p d f$ of $V_{j}$ and $\Gamma_{j}$ given that $s$ was sent ( $s=0$ or 1$)$. The proposed method allows the $j$ th receiver to take on one of $L$ values instead of only two values. This can be done by mapping the $j$ th likelihood ratio, which is a function of $V_{j}$ and $\Gamma_{j}$, using $B$ number of bits $\left(L=2^{B}\right)$. Thus the $j$ th receiver decides $u_{i}$ $=k$ if $\left(v_{j}, \gamma_{j}\right) \in A_{j, k}$, where:

$A_{j k}=\left\{\left(v_{j}, \gamma_{j}\right): t_{j, k-1} \leq \ln \left(\frac{f v_{j}\left(v_{j} \mid 1 \operatorname{sent}, \gamma_{j}\right)}{f v_{j}\left(v_{j} \mid 0 \operatorname{sent}, \gamma_{j}\right)}\right)<t_{j, k}\right\}$

For a given receiver threshold $t_{j}$, if $\ln \left(\frac{f v_{j}\left(v_{j} \mid 1 \text { sent }, \gamma_{j}\right)}{f v_{j}\left(v_{j} \mid 0 \text { sent }, \gamma_{j}\right)}\right)$ is low (or high) enough, the $j$ th receiver decision will take the value 0 (or 1 ) with high probability level and vise versa. We perform this mapping using the following relations:

$u_{j}=2\left(\frac{\ln \left(\frac{f v_{j}\left(v_{j} \mid 1 \operatorname{sent}, \gamma_{j}\right)}{f v_{j}\left(v_{j} \mid 0 \text { sent }, \gamma_{j}\right)}\right)-t_{\min }}{t_{\max }-t_{\min }}\right)^{2} ;$
if $t_{\min } \leq \ln \left(\frac{f v_{j}\left(v_{j} \mid 1 \text { sent }, \gamma_{j}\right)}{f v_{j}\left(v_{j} \mid 0 \text { sent }, \gamma_{j}\right)}\right) \leq t_{j}$

$$
\begin{aligned}
& u_{j}=1-2\left(\frac{\ln \left(\frac{f v_{j}\left(v_{j} \mid 1 \text { sent }, \gamma_{j}\right)}{f v_{j}\left(v_{j} \mid 0 \text { sent }, \gamma_{j}\right)}\right)-t_{\min }}{t_{\max }-t_{\min }}\right)^{2} ; \\
& \text { if } t_{j} \leq \ln \left(\frac{f v_{j}\left(v_{j} \mid 1 \text { sent }, \gamma_{j}\right)}{f v_{j}\left(v_{j} \mid 0 \text { sent }, \gamma_{j}\right)}\right) \leq t_{\max }
\end{aligned}
$$

By this way we ensure that the value of the receiver decision is gradually varies from zero to one according to the difference between the likelihood ratio $\ln \left(\frac{f v_{j}\left(v_{j} \mid 1 \text { sent, } \gamma_{j}\right)}{f v_{j}\left(v_{j} \mid 0 \text { sent }, \gamma_{j}\right)}\right)$ and the receiver threshold $i_{j}$.

The issue of optimum mapping between the likelihood ratio and the receiver decision is not considered here, but simply a uniform mapping is assumed, which is very simple. Thus the values of the receiver decisions in the intervals $[0,1]$ are divided into $L$ uniform step size levels, with $1 / L$ step size value. If the value of the receiver decision falls within the $k$ th interval $(k=1$, $2, \ldots \ldots ., L)$, then the mapped value is taken to be the midpoint of that interval.

\section{Deriving the Combining Fusion Rule of the Digital Central Receiver}

The minimum probability of error (optimum) approach for the central receiver to fuse the individual receiver data is to form the likelihood ratio. If the data in the digital communication channel is equally likely, then the central receiver threshold will be zero. In this case, the global decision for the central receiver is (assuming independent receiver's decisions):

$u_{g}=\left\{\begin{array}{c}1 \text { if } \ln \frac{\operatorname{Prob}\left(u_{1}, u_{2}, \ldots \ldots ., u_{n} \mid 1 \text { sent }\right)}{\operatorname{Prob}\left(u_{1}, u_{2}, \ldots \ldots, u_{n} \mid 0 \text { sent }\right)}>0 \\ 0 \quad \text { othervise }\end{array}\right.$

where, the required probabilities in (10) can be evaluated as: 


$$
\frac{\operatorname{Prob}\left(u_{1}, u_{2}, \ldots \ldots, u_{n} \mid 1 \text { sent }\right)}{\operatorname{Prob}\left(u_{1}, u_{2}, \ldots \ldots, u_{n} \mid 0 \text { sent }\right)}=\coprod_{j=1}^{n} \frac{\operatorname{Prob}\left(u_{j}, \mid 1 \text { sent }\right)}{\operatorname{Prob}\left(u_{j}, \mid 0 \text { sent }\right)}
$$

If we assume that $n_{0}$ receivers decide $0, n_{1}$ receivers decide $u_{j 1}, n_{2}$ receivers decide $u_{j 2}, \ldots, n_{L}$ receivers decide $u_{j L}$ and $n_{+}$receivers decide 1 , then (11) can be rewritten as:

$$
\begin{aligned}
& \coprod_{j=1}^{n} \frac{\operatorname{Prob}\left(u_{j} \mid 1 \text { sent }\right)}{\operatorname{Prob}\left(u_{j} \mid 0 \text { sent }\right)}=\coprod_{j=1}^{n_{0}} \frac{\operatorname{Prob}\left(u_{j}=0 \mid 1 \text { sent }\right)}{\operatorname{Prob}\left(u_{j}=0 \mid 0 \text { sent }\right)} \ldots \\
& \coprod_{j=1}^{n_{L}} \frac{\operatorname{Prob}\left(u_{j}=u_{j L} \mid 1 \text { sent }\right)}{\operatorname{Prob}\left(u_{j}=u_{j L} \mid 0 \text { sent }\right)} \coprod_{j=1}^{n_{+}} \frac{\operatorname{Prob}\left(u_{j}=1 \mid 1 \text { sent }\right)}{\operatorname{Prob}\left(u_{j}=1 \mid 0 \text { sent }\right)}
\end{aligned}
$$

Taking the natural logarithm (monotonic increasing function) of (12) leads to:

$$
\begin{aligned}
& \ln \left(\coprod_{j=1}^{n} \frac{\operatorname{Prob}\left(u_{j} \mid 1 \text { sent }\right)}{\operatorname{Prob}\left(u_{j} \mid 0 \text { sent }\right)}\right)=\sum_{j=1}^{n_{0}} \ln \left(\frac{\operatorname{Prob}\left(u_{j}=0 \mid 1 \text { sent }\right)}{\operatorname{Prob}\left(u_{j}=0 \mid 0 \text { sent }\right)}\right) \\
& +\ldots .+\sum_{j=1}^{n_{+}} \ln \left(\frac{\operatorname{Prob}\left(u_{j}=1 \mid 1 \text { sent }\right)}{\operatorname{Prob}\left(u_{j}=1 \mid 0 \text { sent }\right)}\right)
\end{aligned}
$$

Where:

$$
\begin{aligned}
& \operatorname{Prob}\left(u_{j}=0 \mid \text { ssent }\right) \\
& =\int_{0}^{t_{\min }} f v_{j}\left(v_{j} \mid 1 \text { sent }\right) d v_{j}, j=1,2, \ldots . ., n_{0}, s=0,1 \\
& \operatorname{Prob}\left(u_{j}=u_{j k} \mid \text { ssent }\right)=\int_{t(k-1)}^{t_{k}} f v_{j}\left(v_{j} \mid 1 \text { sent }\right) \\
& d v_{j}, k=1,2, \ldots . ., L, j=1:\left(n_{1}+. .+n_{L}\right), s=0,1 \\
& \operatorname{Prob}\left(u_{j}=1 \mid \text { ssent }\right)=\int_{t \max }^{\infty} f v_{j}\left(v_{j} \mid 1 \text { sent }\right) \\
& d v_{j}, j=1,2, \ldots ., n_{+}, s=0,1
\end{aligned}
$$

When estimates of the receiver $S N R$ 's are available, the probability terms in (14) can be evaluated in terms of the probability density function of the $S N R$ as:

$$
\begin{aligned}
& \operatorname{Prob}\left(u_{j}=u_{j k} \mid \text { ssent }\right)=\int_{\left(v_{j}, \gamma_{j}\right) \in A_{j, k}} f v_{j}\left(v_{j} \mid 1 \text { sent }, \gamma_{j}\right) \\
& d v_{j}, d \gamma_{j}, j=1: n, k=1: L, s=0,1
\end{aligned}
$$

From (4) and (7), the decision rule for the central receiver can be rewritten as:

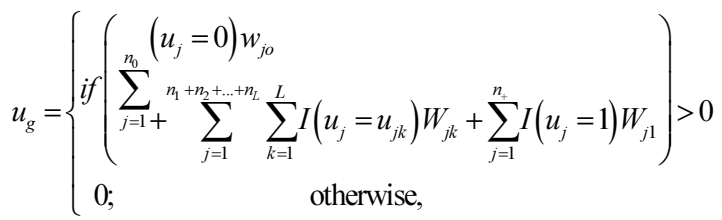

Where:

$$
W_{j k}=\left\{\ln \left(\frac{\operatorname{prob}\left(u_{j}=c \mid 1 \text { sent }\right)}{\operatorname{prob}\left(u_{j}=c \mid 0 \text { sent }\right)}\right), k=1,2, \ldots ., L, c=0,1, u_{j k}\right.
$$

and $I\left(u_{j}=h\right)$ is an indicator function which is unity if $u_{j}=$ $h$ and zero otherwise. From (15) and (17) and by taking into consideration the percentage of the confidence levels for each receiver decision, we can write:

$$
\begin{aligned}
& \int \ln \left(\frac{\int_{\left(v_{j}, \gamma_{j}\right) \in A_{j, 0}} f \Gamma_{j}\left(\gamma_{j}\right) f v_{j}\left(v_{j} \mid 1 \text { sent }, \gamma_{j}\right) d v_{j} d \gamma_{j}}{\int_{\left(v_{j}, \gamma_{j}\right) \in A_{j, k}} f \Gamma_{j}\left(\gamma_{j}\right) f v_{j}\left(v_{j} \mid 0 \operatorname{sent}, \gamma_{j}\right) d v_{j} d \gamma_{j}}\right) \\
& \text { if } u_{j}=0, j=1,2, \ldots, n_{0} \\
& \ln \left(u_{j} \frac{\int_{\left(v_{j}, \gamma_{j}\right) \in A_{j, k}} f \Gamma_{j}\left(\gamma_{j}\right) f v_{j}\left(v_{j} \mid 1 \text { sent }, \gamma_{j}\right) d v_{j} d \gamma_{j}}{\int_{\left(v_{j}, \gamma_{j}\right) \in A_{j, k}} f \Gamma_{j}\left(\gamma_{j}\right) f v_{j}\left(v_{j} \mid 0 \text { sent }, \gamma_{j}\right) d v_{j} d \gamma_{j}}\right)
\end{aligned}
$$

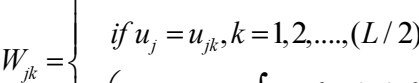

$$
\begin{aligned}
& \ln \left(\left(1-u_{j}\right) \frac{\int_{\left(v_{j}, \gamma_{j}\right) \in A_{j, k}} f \Gamma_{j}\left(\gamma_{j}\right) f v_{j}\left(v_{j} \mid 1 \text { sent }, \gamma_{j}\right) d v_{j} d \gamma_{j}}{\int_{\left(v_{j}, \gamma_{j}\right) \in A_{j, k}} f \Gamma_{j}\left(\gamma_{j}\right) f v_{j}\left(v_{j} \mid 0 \text { sent }, \gamma_{j}\right) d v_{j} d \gamma_{j}}\right) \text {; } \\
& \text { if } u_{j}=u_{j k}, k=(L / 2+1): L \\
& \ln \left(\frac{\int_{\left(v_{j}, \gamma_{j}\right) \in A_{j, 1}} f \Gamma_{j}\left(\gamma_{j}\right) f v_{j}\left(v_{j} \mid 1 \text { sent }, \gamma_{j}\right) d v_{j} d \gamma_{j}}{\int_{\left(v_{j}, \gamma_{j}\right) \in A_{j, 1}} f \Gamma_{j}\left(\gamma_{j}\right) f v_{j}\left(v_{j} \mid 0 \operatorname{sent}, \gamma_{j}\right) d v_{j} d \gamma_{j}}\right) \\
& \text { if } u_{j}=1, j=1,2, \ldots, n_{+}
\end{aligned}
$$

\section{Performance Comparison}

The performance is evaluated in terms of error rate in case of noncoherent FSK in slow Rayleigh fading and additive Gaussian noise. The probability of error of the central receiver is:

$$
\begin{aligned}
& \mathrm{P} e=\operatorname{prob}(0 \text { sent }) \operatorname{prob}(\text { error } \mid 1 \text { sent }) \\
& +\operatorname{prob}(1 \text { sent }) \operatorname{prob}(\text { error } \mid 1 \text { sent })
\end{aligned}
$$

Where:

$$
\begin{aligned}
& \operatorname{prob}(\text { error } \mid 0 \text { sent })=\sum_{u_{1}=0, u_{11}, u_{12}, \ldots \ldots, 1} \ldots \ldots \sum_{u_{n}=0, u_{n 1}, u_{n 2}, \ldots \ldots, 1} \operatorname{prob} \\
& \left(u_{g}=1 \mid u_{1}, \ldots, u_{n}\right) \times \operatorname{prob}\left(u_{1} \mid 1 \text { snet }\right) \ldots . \operatorname{prob}\left(u_{n} \mid 0 \text { snet }\right)
\end{aligned}
$$


and Prob $\left(u_{g}=1 \mid u_{1}, \ldots, u_{n}\right)$ is determined using the central receiver fusion rule (10). Similar equation exists for Prob (error $\mid 1$ sent). In case of slow Rayleigh fading, the probability density function of the signal-to-noise ratio at the $j$ th receiver, $f \Gamma_{j}\left(\gamma_{j}\right)$, will be (Blum, 1999; ElAyadi et al., 1996; Aziz, 2011c; 2009b; 1997):

$$
f \Gamma_{j}\left(\gamma_{j}\right)=\frac{1}{\mu_{j}} \exp \left(-\frac{\gamma_{j}}{\mu_{j}}\right) U\left(\gamma_{j}\right)
$$

where, $\mu_{j}$ is the average signal-to-noise ratio at the $j$ th receiver and:

$$
U(x)=\left\{\begin{array}{l}
1 ; \text { if } x \geq 0 \\
0 ; \text { otherwise }
\end{array}\right.
$$

Using limiting argument and assuming that the two bandpass filters at each receiver have non overlapping passband which are sufficiently separated, we can deduce $f v_{j}\left(v_{j} \mid 0\right.$ sent, $\left.\gamma_{j}\right)$ and $f v_{j}\left(v_{j} \mid 1\right.$ sent, $\left.\gamma_{j}\right)$ as in (Blum, 1999; Willett et al., 2000; Aziz, 1999; Aziz et al., 1997).

To simplify matters, we assume the case of identical receivers (same receiver thresholds). We also assume that an accurate estimate of the signal-to-noise ratio of the observations is available at each individual receiver and the estimate is equal to the true $S N R$. The actual values of the minimum and maximum thresholds depend on the expected noise range. The noise range is taken to be $3 \sigma$, where $\sigma^{2}$ is the average power of the noise. Figure 2 shows a plot of the probability of error versus $S N R$ for different values of number of receivers, assuming two bits for each receiver decision $(B=2)$. The global performance improvement as the number of receivers increases is obvious. Figure 3 compares the global performance of the optimum low resolution approach and the proposed course resolution approach as well as the optimum high resolution approach in case of fifteen identical sensors $(n=15)$ for different $S N R$. The degradation in the performance of the optimum low resolution approach compared to the proposed course resolution and the optimum high resolution approaches is obvious. It is clear from Fig. 3 that the global performance of the optimum high resolution approach is much better than the performance of the optimum low resolution approach for the same $S N R$. The global performance improvement of the proposed approach with $B=2$ is significant. The increment in the global performance improvement in case of the proposed approach decreases as the number of bits per decision increases. The performance of the proposed approach with two or three bits per decision is much better than the performance of the optimum low resolution approach. The performance of the proposed approach with three bits per decision $(B=3)$ is reasonably close to the performance of the optimum high resolution approach. Figure 4 shows the same plots, as in Fig. 3, in case of twenty five identical sensors $(n=25)$ for different $S N R$. It is clear from Fig. 4 that the previous results are also valid. Since the proposed approach achieves a reasonable performance, compared to the performance of the optimum high resolution approach, with few numbers of bits, it reduces cost and complexity of receiver designs considerably.

Figure 5-7 show the plots of the probability of error of the proposed course resolution diversity approach versus $S N R$ for different values of number of bits per receiver decision $(B)$ assuming a fixed value of number of receivers $(n)$. Figure 5 shows the plot of the probability of error versus $S N R$ for $B=1,2,3,4$ and 5, assuming ten receivers $(n=10)$. It is clear from Fig. 5 that the error probability decreases as the number of bits per decision increases. As shown in Fig. 5, there is a significant improvement in the global performance when $B$ increases from 1 to 2 or from 2 to 3 . The global performance improvement is insignificant when $B=4$ or 5. Figure 6 and 7 show the same plots, as in Fig. 5, in case of sixteen $(n=16)$ and twenty two $(n=22)$ receivers, respectively. From Fig. 5-7, it is clear that using three or four bits per decision may be most appropriate. It is impractical to use more than three or four bits per decision to avoid additional hardware cost without significant performance improvement.

The performance of the proposed soft approach is compared to the performance of the course resolution with quality information approach presented in (Thomopoulos et al., 1987) where the local receivers send one or more quality bit information in addition to the individual receiver binary decisions ((Thomopoulos et al., 1987) for the details). The performance of the proposed soft approach is also compared to the performance of the optimum course resolution approach (highest performance) presented in (Lee and Chao, 1989) where the optimum partitioning is derived using the maximum distance criterion ((Lee and Chao, 1989) for the details). The results are shown in Fig. 8 and 9 for the same numbers of bits per decisions $B$. Figure 8 compare the performances assuming that $n=12$ and $B=2$ and Fig. 9 shows the same plots assuming that $n=17$ and $B=3$.

It is clear from Fig. 8 and 9 that the proposed course resolution approach outperforms the course resolution with quality information approach (Thomopoulos et al., 1987) for the same number of bits. Unlike the course resolution with quality information approach, the proposed course resolution approach can be applied easily to non-identical receivers and can be extended easily to any number of bits. Figure 8 and 9 also show that the optimum course resolution approach (Lee and Chao, 1989) has a little performance improvement over the proposed course resolution approach for the same number of bits. 


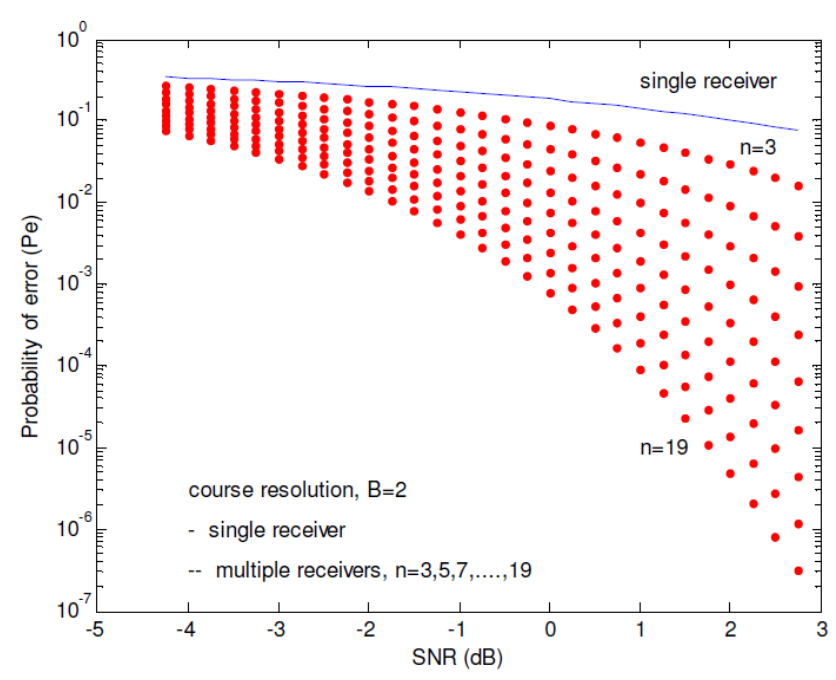

Fig. 2. Error rate performance versus SNR for different values of number of receivers $(n)$

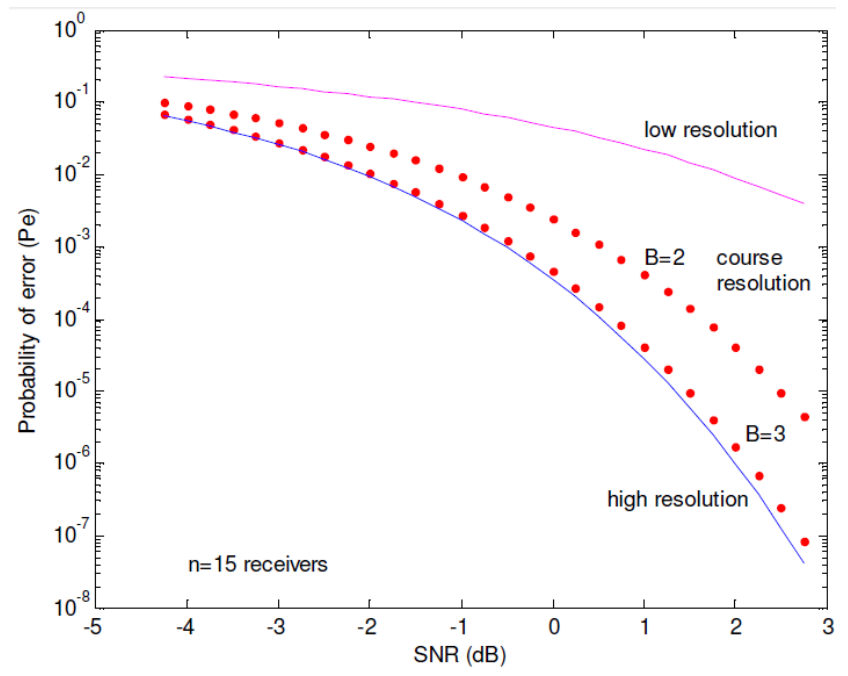

Fig. 3. Comparison of low, course and high resolution error rate performance, $n=15$

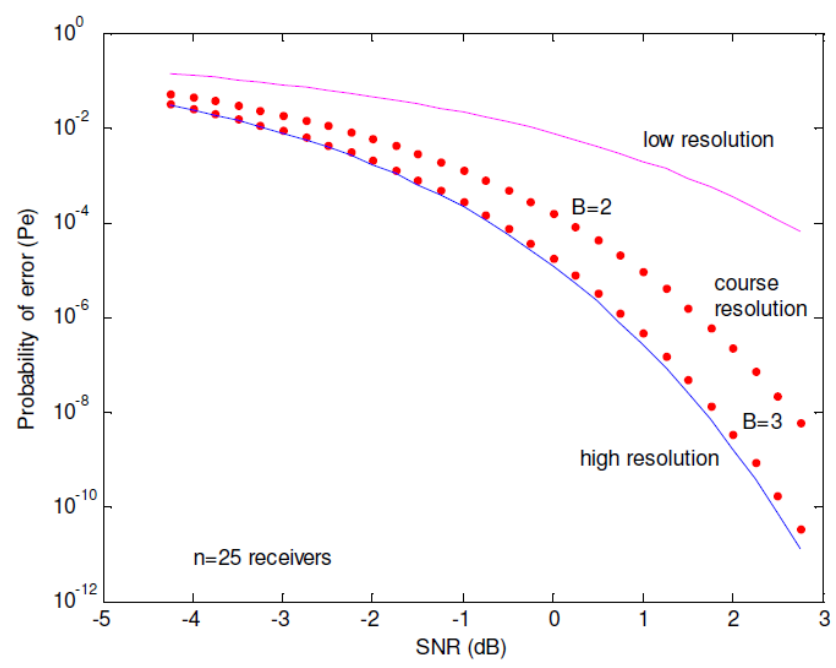

Fig. 4. Comparison of low, course and high resolution error rate performance, $n=25$ 


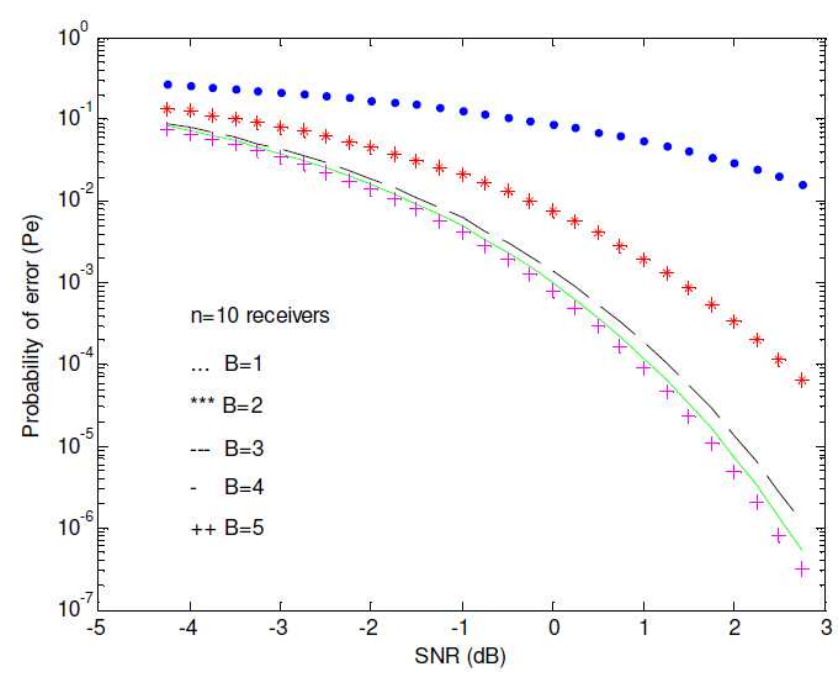

Fig. 5. Comparison of the proposed approach error rate performance for different values of number of bits per receiver decision $(B), n=10$

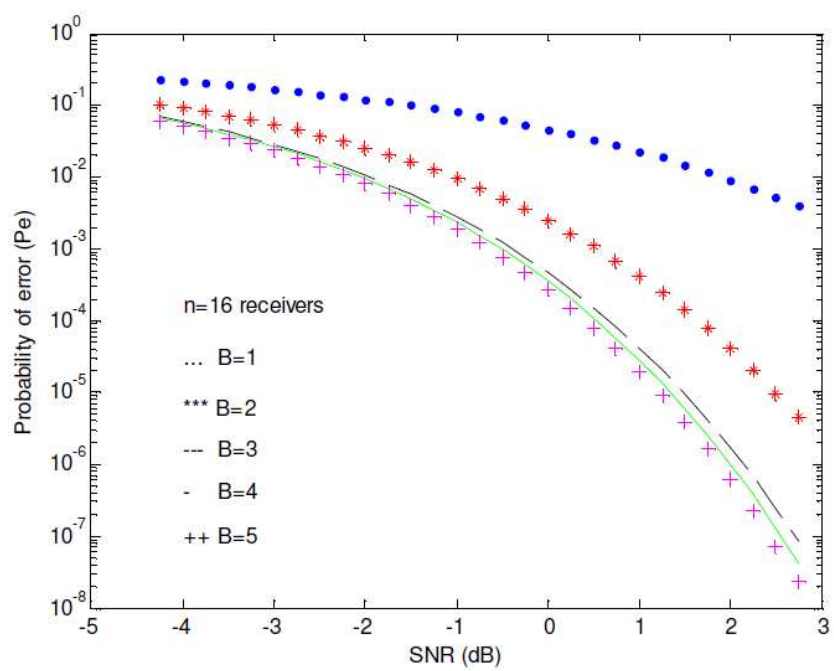

Fig. 6. Comparison of the proposed approach error rate performance for different values of number of bits per receiver decision $(B), n=16$

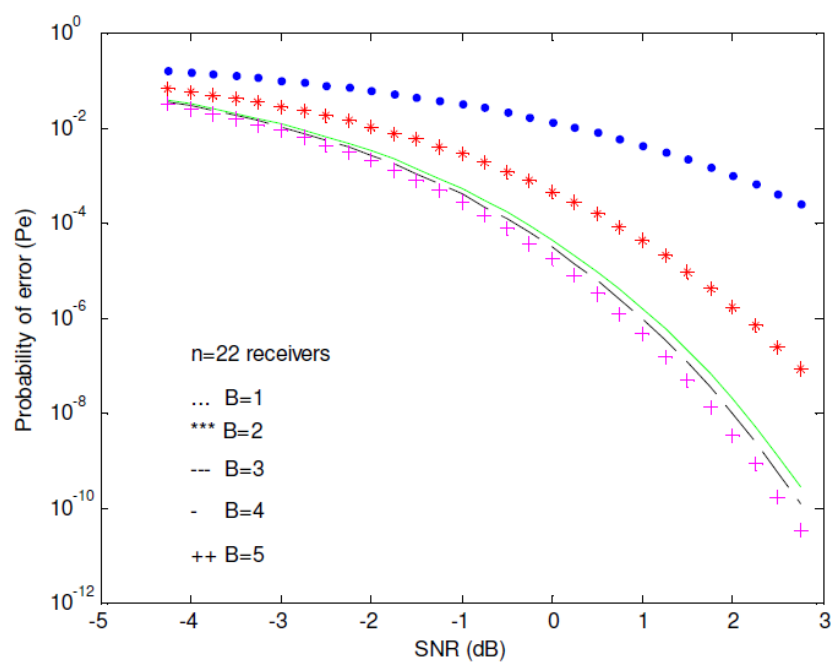

Fig. 7. Comparison of the proposed approach error rate performance for different values of number of bits per receiver decision $(B), n=22$ 


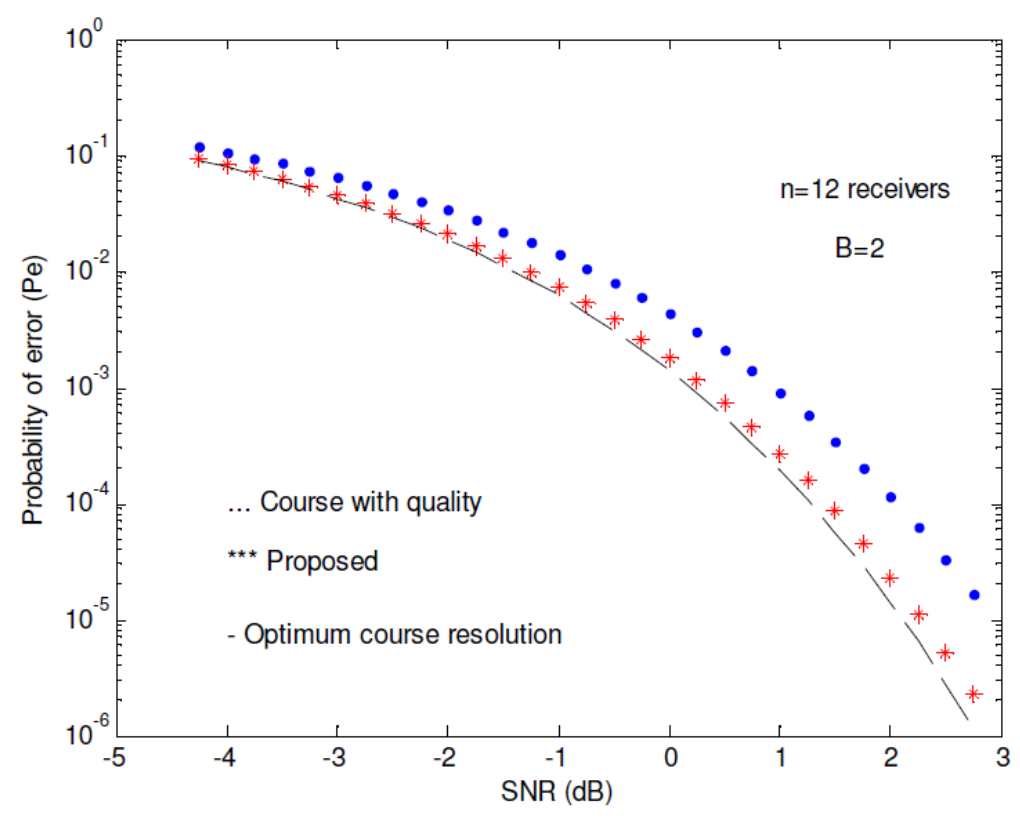

Fig. 8. Comparison of the proposed approach, course resolution with quality information approach and the optimum course resolution approach for $n=12$ and $B=2$

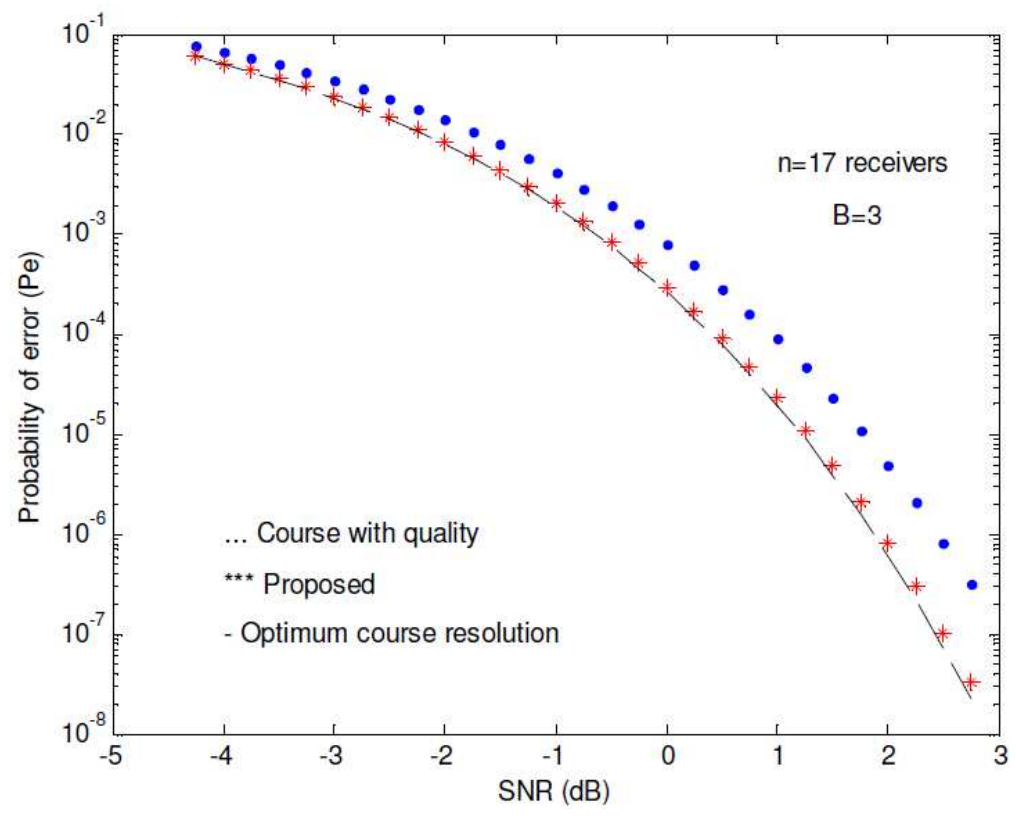

Fig. 9. Comparison of the proposed approach, course resolution with quality information approach and the optimum course resolution approach for $n=17$ and $B=3$

However, unlike the optimum course resolution approach, which requires the derivatives of the functional relationships between the individual receiver error probabilities and the individual receiver thresholds, the proposed course resolution approach does not require these relationships. It is also clear from Fig. 8 and 9 that the performance improvement of the optimum course resolution approach over the proposed course resolution approach decreases as the number of receivers and the number of bits per decisions increase.

Compared to other course resolution approaches, the novelty of the proposed course resolution approach is that each receiver generates a course decision value between 0 and 1 according to the difference between the receiver's likelihood ratio and the receiver's threshold. Each receiver's course decision represents its 
degree of reliability on that decision. Unlike the published course resolution approaches, the combining rule of the proposed course resolution approach fuses reliability terms weighted by confidence levels to derive the decision of the central receiver. From the previous examples, it is clear that the proposed course resolution approach is very efficient. In addition, the proposed course resolution approach has the following advantages: (1) it can be applied easily to any number of non-identical and identical receivers, (2) it can be extended easily to any number of bits, (3) it can be applied easily to any type of distributed observations, (4) does not require the derivatives of the functional relationships between the individual receiver error probabilities and the individual receiver thresholds and (5) the computations do not grow exponentially either as the number of bits or the number of sensors increases. The results of these advantages are the reduction of complexity and the feasibility for real-time processing.

\section{Conclusion}

An efficient course resolution multiple-receiver approach for reception of slow fading signals in digital wireless communication systems has been proposed. The case of noncoherent FSK, in case of slow Rayleigh fading and additive Gaussian noise affecting the observations at each individual receiver, has been considered. At each individual receiver, course data is made. All receiver data is sent to a central receiver where final global data is developed which indicates which symbol was transmitted. Performance evaluation of the proposed approach has been provided and compared to that of the optimum low and high resolution approaches. It has been shown that the proposed approach reduces cost and complexity of receiver designs considerably. It has been shown that the use of course resolution approach can improve the performance of the central receiver combiner. It has been shown that the performance of the proposed approach is better than the performance of the optimum low resolution approach and is reasonably close to the performance of the high resolution approach using only few numbers of bits. Thus it is impractical to use more than three or four bits per receiver decision to avoid additional hardware construction without significant performance improvement.

\section{Acknowledgement}

The main author sincerely thanks Professors M.H. ElAyadi and E.I. Eweda. They played a big part in his development during his study in the Military Technical College, Cairo, Egypt.

\section{Author's Contributions}

Ashraf M. Aziz: Developed the research idea and organized the study. Coordinated the overall framework and participated in all simulations, discussion, data-analysis as well as contributed to the writing of the manuscript.

Mohamed A. Abdel-Rahman: Organized the study. Coordinated the overall framework and participated in all simulations, discussion, data-analysis as well as contributed to the writing of the manuscript.

Saeed A. Al-Ghamdi: Organized the study. Coordinated the overall framework and participated in all simulations, discussion, data-analysis as well as contributed to the writing of the manuscript.

\section{Ethics}

This article is original and contains unpublished material. The corresponding author confirms that all of the other authors have read and approved the manuscript and no ethical issues involved.

\section{References}

Aziz, A.M., 1997. Multiple-sensor distributed detection systems with data fusion. Proceedings of the 13th International Conference on Digital Signal Processing, Jul. 2-4, IEEE Xplore Press, Santorini, pp: 1031-1034. DOI: 10.1109/ICDSP.1997.628540

Aziz, A.M., 1999. New data fusion algorithms for distributed multi-sensor multi-target environments. PhD Thesis, Department of Electrical Computer Engineering.

Aziz, A.M., 2009a. Effects of fuzzy membership function shapes on clustering performance in multisensor-multitarget Data fusion systems. Proceedings of the IEEE International Conference on Fuzzy Systems, Aug. 20-24, IEEE Xplore Press, Jeju Island, pp: 1839-1844.

DOI: 10.1109/FUZZY.2009.5277313

Aziz, A.M., 2009b. A new all-neighbor fuzzy association technique for multitarget tracking in a cluttered environment. Proceedings of the IEEE International Conference on Fuzzy Systems, Aug. 20-24, IEEE Xplore Press, Jeju Island, pp: 1767-1772. DOI: 10.1109/FUZZY.2009.5277347

Aziz, A.M., 2011a. A soft-decision fusion approach for multiple-sensor distributed binary detection systems. IEEE Trans. Aerospace Electronic Syst., 47: 2208-2216. DOI: 10.1109/TAES.2011.5937293

Aziz, A.M., 2011b. A fuzzy approach for multiplereceiver digital communication systems with data fusion. Int. J. Electronics Commun., 65: 406-412. DOI: $10.1016 /$ j.aeue.2010.05.006 
Aziz, A.M., 2011c. A novel all-neighbor fuzzy association approach for multitarget tracking in a cluttered environment. Signal Process., 91: 2001-2015. DOI: 10.1016/j.sigpro.2011.03.007

Aziz, A.M., 2013. A new nearest-neighbor association approach based on fuzzy clustering. Aerospace Sci. Technol., 26: 87-97. DOI: 10.1016/j.ast.2012.02.017

Aziz, A.M., 2014a. A joint possibilistic data association technique for tracking multiple targets in a cluttered environment. Inform. Sci., 280: 239-260.

DOI: $10.1016 /$ j.ins.2014.04.055

Aziz, A.M., 2014b. A new multiple decisions fusion rule for targets detection in multiple sensors distributed detection systems with data fusion. Inform. Fusion, 18: 175-186. DOI: 10.1016/j.inffus.2013.09.002

Aziz, A.M., 2014c. A new adaptive decentralized soft decision combining rule for distributed sensor systems with data fusion. Inform. Sci., 256: 197-210. DOI: 10.1016/j.ins.2013.09.031

Aziz, A.M., A.M. El-Bakly, M.H.A. Azeem and G.M.A. Hamid, 2011. A new soft-fusion approach for multiple-receiver wireless communication systems. Electronics Telecommun. Res. Institute J., 33: 310-319. DOI: 10.4218/etrij.11.0110.0282

Aziz, A.M., M. Tummala and R. Cristi, 1997. Optimal data fusion strategies using multiple-sensor detection systems. Proceedings of the 31 st Asilomar Conference on Signal, Systems and Computers, Nov. 2-5, IEEE Xplore Press, Pacific Grove, pp: 941-945. DOI: 10.1109/ACSSC.1997.680582

Blum, R.S., 1999. Distributed detection for diversity reception of fading signals in noise. IEEE Trans. Inform. Theory, 45: 158-164.

DOI: $10.1109 / 18.746782$

Chau, Y. and J. Sun, 1996. Diversity with distributed decisions combining for direct-sequence CDMA in a shadowed rician-fading land-mobile satellite channel. IEEE Trans. Vehicular Technol., 45: 237-247. DOI: $10.1109 / 25.492847$

Chen, Y. and C. Tellambura, 2004. Distribution functions of selection combiner output in equally correlated Rayleigh, rician and nakagami-m fading channels. IEEE Trans. Commun., 52: 1948-1956. DOI: 10.1109/TCOMM.2004.836596

ElAyadi, M.H., A.M. Aziz and A. ElBasiouny, 1996. An algorithm for global optimization of distributed multiple-sensor detection systems using NeymanPearson strategy. Signal Process., 52: 137-145. DOI: 10.1016/0165-1684(96)00038-2
Hu, J. and R.S. Blum, 2002. Application of distributed signal detection to multiuser communication systems. IEEE Trans. Aerospace Electronic Syst., 38: 1220-1229. DOI: 10.1109/TAES.2002.1145745

Kot, A.D. and C. Leung, 2003. Optimal partial decision combining in diversity systems. IEEE Trans. Commun., 51: 1927-1938. DOI: 10.1109/26.57496

Lee, C.C. and J.J. Chao, 1989. Optimum local decision space partitioning for distributed detection. IEEE Trans. Aerospace Electronic Syst., 25: 536-543. DOI: $10.1109 / 7.32086$

Mirjalily, G., Z.Q. Luo, T.N. Davidson and E. Bosse, 2003. Blind adaptive decision fusion for distributed detection. IEEE Trans. Aerospace Electronic Syst., 39: 34-52. DOI: 10.1109/TAES.2003.1188892

Sendonaris, A., E. Erkip and B. Aazhang, 2007. User cooperation diversity. Part I. system description. IEEE Trans. Commun., 55: 887-811.

DOI: 10.1109/TCOMM.2003.818096

Shin, W.Y. and Y.H. Lee, 2008. Diversitymultiplexing tradeoff and outage performance for Rician Mimo channels. IEEE Trans. Inform. Theory, 54: 1186-1196. DOI: 10.1109/TIT.2007.915884

Simon, M.K. and M.S. Alouini, 2005. Digital Communication over Fading Channels. 2nd Edn., John Wiley and Sons, New Jersey, ISBN-10: 0471715239, pp: 936.

Thomopoulos, S., R. Viswanathan and D. Bougoulias, 1987. Optimal decision fusion in multiple sensor systems. IEEE Trans. Aerospace Electronic Syst., 23: 644-653. DOI: 10.1109/TAES.1987.310858

Tse, D., P. Viswanath and L. Zheng, 2004. Diversitymultiplexing tradeoff in multiple-access channels. IEEE Trans. Inform. Theory, 50: 1859-1874. DOI: 10.1109 /TIT.2004.833347

Viswanathan, R. and P.K. Varshney, 1997. Distributed detection with multiple sensors I. Fundamentals. Proc. IEEE, 85: 54-63. DOI: 10.1109/5.554208

Wen, Q. and J.A. Riteey, 1994. Spatial diversity equalization applied to underwater communications. IEEE J. Oceanic Eng., 19: 227-241. DOI: $10.1109 / 48.286646$

Willett, P., P.F. Swaszek and R.S. Blum, 2000. The good, bad and ugly: Distributed detection of a known signal in dependent Gaussian noise. IEEE Trans. Signal Process., 48: 3266-3279. DOI: $10.1109 / 78.886990$ 